\title{
Nota
}

\section{ESTOQUES DE CARBONO E NITROGÊNIO NO SOLO CULTIVADO COM MAMONA NA CAATINGA ${ }^{(1)}$}

\author{
Felipe José Cury Fracetto ${ }^{(2)}$, Giselle Gomes Monteiro Fracetto ${ }^{(3)}$, Carlos Clemente \\ Cerri $^{(4)}$, Brigitte Josefine Feigl ${ }^{(5)} \&$ Marcos Siqueira Neto ${ }^{(6)}$
}

\begin{abstract}
RESUMO
Grande parte da produção brasileira de mamona encontra-se no Nordeste, como opção de cultura para a região semiárida no bioma Caatinga. $O$ objetivo deste estudo foi avaliar as alterações nos estoques de $\mathrm{C}$ e $\mathrm{N}$ devido à mudança de uso do solo de vegetação natural para o cultivo de mamona no bioma Caatinga. Este trabalho foi realizado na Fazenda Floresta, no município de Irecê, no centro-norte baiano. $O$ clima da região é do tipo BSwh (Köppen) - clima semiárido de altitude. O solo foi classificado em Latossolo Vermelho de textura argilosa. As situações avaliadas foram: três áreas cultivadas com mamona com diferentes tempos de implantação: (i) com 10 anos, (ii) com 20 anos e (iii) com 50 anos; e uma área de referência (vegetação nativa de Caatinga) contígua às situações avaliadas. Os estoques de $\mathrm{C} e$ $\mathrm{N}$ foram determinados em amostras de solo coletadas em cinco minitrincheiras, nas camadas de 0-5, 5-10, 10-20 e 20-30 cm. Os resultados mostraram que o constante aporte de resíduos vegetais na Caatinga promoveu a manutenção dos estoques de $\mathrm{C}\left(90 \mathrm{Mg} \mathrm{ha}^{-1}\right)$ e $\mathrm{N}\left(10 \mathrm{Mg} \mathrm{ha}^{-1}\right)$ para a camada de 0-30 $\mathrm{cm}$. A mudança de uso da terra para o cultivo da mamona ocasiona redução em aproximadamente $50 \%$ nos estoques de $\mathrm{C}$ e $\mathrm{N}$ do solo em relação à vegetação nativa nos primeiros 10 anos de implantação da cultura. A meia-vida da matéria orgânica do solo (MOS) calculada para essa situação na região do semiárido foi de 4,7 anos. $O$ fator de emissão de $C$ do solo, devido à mudança de uso da terra após 20 anos, conforme proposto pelo método do

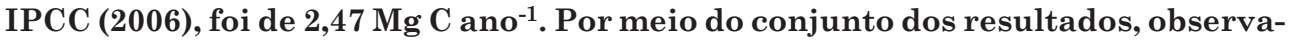
se a fragilidade do solo do bioma Caatinga no que se refere à perda de MOS devido à mudança de uso da terra.
\end{abstract}

Termos de indexação: semiárido, mudança de uso da terra, efeito estufa.

(1) Parte da dissertação de mestrado do primeiro autor. Recebido para publicação em 14 de outubro de 2011 e aprovado em 24 de julho de 2012 .

(2) Doutorando do Laboratório de Biogeoquímica Ambiental. Centro de Energia Nuclear na Agricultura, Universidade de São Paulo - CENA/USP, Caixa Postal 96. CEP 13400-970 Piracicaba (SP). E-mail: fracetto@esalq.usp.br.

(3) Doutora em Microbiologia Agrícola pelo Laboratório de Microbiologia Molecular, ESALQ/USP. E-mail: giselleih@yahoo.com.br.

(4) Professor Titular do Laboratório de Biogeoquímica Ambiental, CENA/USP. E-mail: cerri@cena.usp.br.

(5) Professora Doutora do Laboratório de Biogeoquímica Ambiental, CENA/USP. E-mail: beduardo@cena.usp.br.

(6) Pós-Doutorando do Laboratório de Biogeoquímica Ambiental, CENA/USP. E-mail: msiqueir@gmail.com. 


\title{
SUMMARY: CARBON AND NITROGEN STOCKS IN SOIL UNDER CASTOR BEAN IN THE SEMI-ARID CAATINGA OF BRAZIL
}

\begin{abstract}
Much of the Brazilian castor bean (Ricinus communis L.) production is concentrated in the semi-arid region (Caatinga biome) of the Northeast, as an alternative crop. The purpose of this study was to evaluate changes in $C$ and $N$ pools due to land use change in the Caatinga biome by castor bean production. The study was conducted on the Fazenda Floresta in the municipality of Irecê (Mid -North region of Bahia, Brazil). The climate is type BSwh (Koppen) - Semi-arid climate. The soil was classified as clayey Oxisol. Three areas under castor bean were evaluated after different cultivation periods i) after 10 years; ii) after 20 years, and iii) after 50 years, and a reference area (native vegetation of the Caatinga) compared with the above situations. $C$ and $N$ stocks were determined in soil samples with five replications (layers 0-5, 5-10, 10-20 and 20-30 cm). The results showed that the constant plant residue input in the native vegetation ensured the maintenance of the $C$ stocks $\left(90 \mathrm{Mg} \mathrm{ha}^{-1}\right)$ and $\mathrm{N}$ stocks (10 $\left.\mathrm{Mg} \mathrm{ha}^{-1}\right)$. The land use change to castor bean production resulted in a reduction of about $50 \%$ in soil $C$ and $N$ stocks compared to Caatinga, in the first 10 years of crop establishment. The half-life of SOM of the semi-arid region was $4.7 \mathrm{yr}$. The C emission factor from soil, due to land use change after 20 years as proposed by the IPCC methodology was

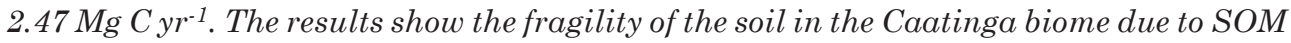
loss caused by land use changes.
\end{abstract}

Index terms: brazilian semi-arid region, land use change, greenhouse effect.

\section{INTRODUÇÃO}

O aumento da mudança de uso do solo com a redução da cobertura da vegetação nativa no semiárido nordestino, assim como nos demais biomas brasileiros, tem levado à degradação dos recursos naturais, em especial no tocante à manutenção da fertilidade natural e redução da matéria orgânica do solo (MOS) (Menezes \& Sampaio, 2002). Além disso, na Caatinga, os problemas de ordem socioambiental são agravados pelas limitações impostas pela escassez de água (PaesSilva, 2002). Por outro lado, Sampaio \& Salcedo (1997) afirmam que, apesar da fragilidade natural desse ecossistema, os mesmos fatores que causam suas limitações conferem certa resistência às perturbações antrópicas às quais ele está frequentemente submetido.

A mamona (Ricinus communis L.) tem sido utilizada como alternativa de cultura e renda no Nordeste, alterando a paisagem, as condições do solo (Brasil, 2002) e a vida dos pequenos agricultores (César \& Batalha, 2010). A maior parte da produção brasileira de mamona está disseminada na região Nordeste e situa-se em sua quase totalidade na Caatinga, cujas condições climáticas podem ser contornadas por meio de variedades resistentes, o que não alteraria drasticamente o seu desenvolvimento (Embrapa, 2007). O sistema apresenta baixo custo de produção e é praticado por pequenos produtores com intensivo emprego de mão de obra, utilizando poucos insumos e podendo ser consorciado com outras culturas (Freitas \& Fredo, 2005)

O óleo é o mais importante constituinte da semente - conhecido como óleo de rícino. Ele é extraído pela prensagem das sementes e contém aproximadamente
$90 \%$ de triglicerídeos derivados do ácido ricinoleico. A produtividade média de mamona em sementes no Recôncavo Baiano é de $800 \mathrm{~kg} \mathrm{ha}^{-1}$, e cada kg de cacho pode render até $800 \mathrm{~mL}$ de óleo (IBGE, 2010).

De acordo com Savy Filho \& Benzatto (1983), o mais tradicional e importante subproduto da mamona é a torta, formada da moagem dos cachos juntamente com restos dos frutos depois da prensagem. O alto teor de proteína torna-a atraente para alimentação animal, porém a presença de compostos tóxicos de difícil eliminação tem tornado inviável essa alternativa. Devido à inexistência de método seguro para sua destoxicação, a torta tem sido utilizada predominantemente como adubo orgânico em culturas de café, milho, feijão, entre outras, e como matériaprima para a produção de aminoácidos, plásticos biodegradáveis, colas, inseticidas, além de servir como controlador da atividade de nematoides no solo (Severino et al., 2004).

Para extração do óleo e obtenção da torta de mamona, é necessário aumento na plantação da mamoneira, ou seja, a mudança de uso do solo da Caatinga no Nordeste brasileiro; por outro lado, é sabido que a MOS contida nos solos sob vegetação nativa sofre modificações quando esses sistemas são convertidos em uso agrícola. Dependendo do sistema de manejo do solo, os estoques podem permanecer iguais, aumentar ou diminuir em relação ao sistema natural (Cerri et al., 2009).

Atualmente, o carbono (C) e o nitrogênio (N) frequentemente têm sido utilizados como indicadores da qualidade do solo, tanto em sistemas agrícolas como em áreas de vegetação nativa (Jansen, 2005). No entanto, alguns fatores de ordem ambiental dificultam 
a realização desses estudos, uma vez que o conhecimento sobre as propriedades edafoclimáticas é limitado, havendo alta variabilidade dos fatores que controlam o ciclo do $\mathrm{C}$ e $\mathrm{N}$-orgânico em uma única unidade de solo (Bernoux et al., 2002).

Em estudos realizados nos diferentes biomas brasileiros, de maneira geral, os autores demonstraram haver relação entre o teor de $\mathrm{C}$ e $\mathrm{N}$ do solo e o tipo de vegetação (Siqueira Neto et al., 2009, 2010; Maia et al., 2009, 2010; Frazão et al., 2010). Especificamente para o bioma Caatinga, os autores discutem tanto ganhos como perdas nos estoques de $\mathrm{C}$ e $\mathrm{N}$ nos diferentes usos do solo (Maia et al., 2006, 2007; Assis et al., 2010; Sousa et al., 2012), sem que haja consenso sobre a dinâmica da MOS em função da mudança de uso. Além disso, inexistem informações em solos cultivados com mamona, principalmente devido às especificidades no manejo dessa cultura. Com base no exposto, o objetivo deste estudo foi avaliar as alterações nos estoques de $\mathrm{C} \mathrm{e} \mathrm{N}$ devido à mudança de uso do solo de vegetação nativa no bioma Caatinga para o cultivo de mamona.

\section{MATERIAL \& MÉTODOS}

As áreas de estudo estão localizadas na Fazenda Floresta, no município de Irecê, no centro-norte baiano

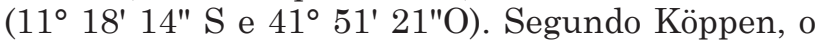
clima da região é do tipo BSwh - clima semiárido (Polígono das Secas) de altitude $(722 \mathrm{~m})$, com pluviosidade média anual de $642 \mathrm{~mm}$, sendo $80 \%$ das chuvas concentradas entre novembro e março, com acentuado déficit hídrico entre abril e outubro. A temperatura média anual é de $23,7^{\circ} \mathrm{C}$, variando entre 15,1 e $30,9^{\circ} \mathrm{C}$. O solo foi classificado em Latossolo Vermelho eutrófico textura argilosa (Embrapa, 2006).

Nessa região, as cultivares de mamona mais utilizadas são a BRS-Nordestina e a BRS-Paraguaçu. Essas espécies apresentam boa resistência a pragas e doenças e produzem maior quantidade de óleo.

Na Fazenda Floresta, a cultura da mamona é conduzida em sistema de produção manual, sem preparo do solo, uso de adubação ou outros insumos agrícolas. A mudança de uso da terra teve início com o desmatamento das primeiras áreas de Caatinga, em 1960, para plantio da mamona intercalada nas entrelinhas com milho. Os espaçamentos para cultivo da mamona são de $2 \mathrm{~m}$ entre plantas e 4,5-6,0 m entre linhas.

Geralmente, em abril ocorre a maturação dos racemos (cachos), com a colheita manual realizada em julho-agosto; durante os meses de maturação e colheita dos racemos, ocorre perda das folhas. Em outubro, é feita uma poda drástica, quando se retira praticamente toda a fitomassa epígea da planta. Em razão da presença de compostos fitotóxicos para a própria cultura, que podem ser liberados durante a decomposição do material vegetal, todos os resíduos vegetais são retirados da área de cultivo e amontoados em outra área da propriedade, para secagem natural e posterior queima.

Dentro da fazenda, três situações de cultivo de mamona com diferentes tempos de implantação foram identificadas, nas quais a mudança de uso do solo foi feita diretamente da vegetação nativa (Caatinga): uma área de 4,55 ha cultivada com mamona desde 1960 (Mamona 50); uma área de 5,20 ha cultivada com mamona desde 1988 (Mamona 20); e uma área de 4,83 ha cultivada com mamona desde 1998 (Mamona 10). Além dessas áreas, foi amostrada uma área de referência sob vegetação nativa (Caatinga), situada imediatamente ao lado das áreas de cultivo.

Para amostragem do solo, foi demarcada uma área com 1 ha na porção central de cada situação. Coletaram-se amostras de solo em cinco trincheiras até $30 \mathrm{~cm}$ de profundidade, seccionadas nas camadas de 0-5, 5-10, 10-20 e 20-30 cm. Em todas as camadas, foram coletadas amostras indeformadas pelo método do anel volumétrico, com um cilindro de aço inox de 5 x $5 \mathrm{~cm}\left(99,4 \mathrm{~cm}^{3}\right)$.

No Laboratório de Biogeoquímica Ambiental (CENA/USP), em Piracicaba (SP), as amostras de solo foram secas ao ar, destorroadas manualmente e peneiradas a $2 \mathrm{~mm}$. Para breve caracterização do solo, utilizou-se uma amostra de solo composta das cinco repetições, coletada na camada de $0-20 \mathrm{~cm}$, na área de Caatinga. $\mathrm{O} \mathrm{pH}$ em água foi determinado usando um extrato com relação 1:2,5 (solo:água); o valor obtido foi de 6,4. Quanto à granulometria, o teor de areia determinado por peneiramento úmido foi de $347 \mathrm{~g} \mathrm{~kg}^{-1}$, enquanto os de argila e silte, determinados pelo método do densímetro, foram de 546 e $107 \mathrm{~g} \mathrm{~kg}^{-1}$, respectivamente.

No tocante à densidade do solo, foi determinada a massa total amostrada nos cilindros, sendo em seguida retirada uma porção de $5 \mathrm{~g}$, para se determinar a umidade gravimétrica. Com base no peso das amostras secas e no volume do cilindro, calculou-se o valor da densidade do solo (Ds), segundo Blake \& Hartge (1986).

Para os teores de $\mathrm{C}$ e N, as amostras foram moídas e passadas em peneiras de 100 mesh $(0,149 \mathrm{~mm})$. Os teores de $\mathrm{C}$ e $\mathrm{N}$ totais foram determinados por espectroscopia no infravermelho próximo, no equipamento Antaris ${ }^{\circledR}$ MDS FT-NIR. Os estoques de $\mathrm{C}$ e $\mathrm{N}$ foram calculados a partir dos valores de $\mathrm{C}$ e $\mathrm{N}$, dos valores da densidade do solo e das camadas de solos amostradas (Equação 1) (Bernoux et al., 1998):

$$
\mathrm{E}=\mathrm{Ds} \mathrm{A} \mathrm{Q}
$$

em que E é o estoque de $\mathrm{C}$ ou $\mathrm{N}$ do solo convertido em hectare $\left(\mathrm{Mg} \mathrm{ha}^{-1}\right)$; Ds, a densidade do solo $\left(\mathrm{g} \mathrm{cm}^{-3}\right) ; \mathrm{A}$, a espessura da camada amostrada $(\mathrm{cm})$; e Q, o teor de $\mathrm{C}$ ou $\mathrm{N}$ do solo (\%).

Contudo, como neste estudo o objetivo foi comparar situações com diferentes tempos de implantação do 
cultivo da mamona, e uma vez que as áreas amostradas apresentaram valores significativamente diferentes na densidade do solo, optou-se pela correção do estoque de $\mathrm{C}$ e $\mathrm{N}$ do solo por uma massa fixa, ou seja, considerando uma densidade de solo igual a $1 \mathrm{~g}$ $\mathrm{cm}^{-3}$, seguindo os cálculos apresentados por Sisti et al. (2004) (Equação 2):

$$
\mathrm{E}_{\mathrm{c}}=\Sigma_{\mathrm{n}-\mathrm{i}} \mathrm{E}+\left\{\left[\mathrm{M}_{\mathrm{ai}}-\left(\Sigma_{\mathrm{n}} \mathrm{M}_{\mathrm{a}}-\Sigma_{\mathrm{n}} \mathrm{M}_{\mathrm{r}}\right)\right] \mathrm{Q}_{\mathrm{i}}\right\}
$$

em que $\mathrm{E}_{\mathrm{c}}$ é o estoque corrigido de $\mathrm{C}$ ou $\mathrm{N}$ pela massa de solo $\left(\mathrm{Mg} \mathrm{ha}^{-1}\right) ; \Sigma_{\mathrm{n}-\mathrm{i}} \mathrm{E}$ é o somatório dos estoques das camadas, sem a última camada amostrada; $\mathrm{M}_{\mathrm{ai}}$ é a massa de solo da última camada de solo amostrado; $\Sigma_{\mathrm{n}} \mathrm{M}_{\mathrm{a}}$ é o somatório da massa total de solo amostrada; $\Sigma_{\mathrm{n}} \mathrm{M}_{\mathrm{r}}$ é o somatório da massa de solo de referência; e $\mathrm{Q}_{\mathrm{i}}$ é o teor de $\mathrm{C}$ ou $\mathrm{N}$ na última camada amostrada.

Após a correção dos estoques de C pela massa de solo amostrada, foi possível determinar a variação do estoque de $\mathrm{C}$ no solo conforme o método proposto no volume 4 do Guia para Inventários Nacionais de Gases de Efeito Estufa (IPCC, 2006), que recomenda a utilização de resultados obtidos nos últimos 20 anos para efetuar os inventários nacionais (Equação 3):

$$
\Delta C=\frac{\sum_{\mathrm{h}=1}\left(C_{f}(h)-\mathrm{C}_{i}(h)\right)}{T}
$$

em que $\Delta C$ é a variação do estoque de $\mathrm{C}$ do solo $(\mathrm{Mg}$ ha $\left.^{-1} \mathrm{ano}^{-1}\right) ; h$ é considerado o sistema de manejo avaliado; $C_{f}(h)$ é o estoque de $\mathrm{C}$ no tempo final $(\mathrm{Mg} \mathrm{C})$ no último ano do inventário para o sistema $\mathrm{h}$ avaliado; $C_{i}(h)$ é o estoque de $\mathrm{C}$ no tempo inicial $(\mathrm{Mg} \mathrm{C})$ na área de referência; e $T$ é o tempo (anos).

Os dados obtidos para cada camada de solo em cada situação amostrada (áreas) foram submetidos ao teste de Kolmogorov-Smirnov $(p=0,05)$ para avaliação da normalidade de distribuição. Por não apresentarem distribuição normal, uma vez que foram amostrados diferentes sítios topográficos para a comparação entre as médias, utilizou-se o teste para múltipla comparação Student Newman-Keuls - teste de SNK $(p<0,05)$. Nas regressões foi aplicado o teste $t(p<0,01$ ou 0,05$)$. As análises foram feitas utilizando o programa SAS (2003).

\section{RESULTADOS \& DISCUSSÃO}

Os valores da densidade do solo (Ds) foram bastante variáveis em todas as camadas nas situações avaliadas (Quadro 1). Na área de Caatinga, observou-se decréscimo nos valores da Ds com o aumento da profundidade, com diferença significativa $(p<0,05)$ apenas na camada superficial. De acordo com Streck et al. (2004), altos valores na densidade encontrada nessa condição são considerados aceitáveis em razão do não revolvimento e da estabilidade de agregados em função das características desse solo, que favorece a compactação superficial.
Nas áreas cultivadas com mamona não foi verificado comportamento comum entre as diferentes idades de implantação para os valores da Ds; contudo, pode-se observar aumento nos valores para a camada de 10-20 cm em relação às demais ( $p<0,05)$.

No conjunto dos resultados, os valores observados variaram entre 0,8 e $1,3 \mathrm{~g} \mathrm{~cm}^{-3}(\mathrm{p}<0,05)$. Dessa forma, para a correta comparação entre as áreas foi necessário fazer a correção dos estoques de $\mathrm{C}$ e $\mathrm{N}$ do solo.

Os maiores teores de $\mathrm{C}$ e $\mathrm{N}$ foram encontrados na Caatinga para todas as camadas amostradas. Nessa situação, os teores de $\mathrm{C}$ decrescem $(\mathrm{p}<0,05)$ com o aumento da profundidade (Quadro 1). Esse comportamento é típico de situação sob vegetação nativa, pois o aporte de resíduos vegetais na superfície do solo promove a decomposição lenta e gradual, a qual garante a constante incorporação de material orgânico no solo. Contudo, essa situação não foi regra para os teores de N, o que, provavelmente, se deve ao tipo de resíduo vegetal incorporado. Por se tratar de vegetação de Caatinga, o aporte de resíduos é pobre em N (Severino et al., 2004), pois, nas condições do semiárido, a ausência de umidade ocasiona menor atividade microbiana na superfície do solo (Xavier et al., 2006); consequentemente, a ciclagem interna do $\mathrm{N}$ na planta deve ser mais eficiente (Galvão et al., 2005) em relação à das florestas úmidas, onde a ciclagem do elemento ocorre na superfície do solo, com a decomposição e reassimilação dos elementos (Menezes et al., 2002).

Da mesma forma, o maior conteúdo de $\mathrm{C}$ na camada superficial do solo pode estar relacionado à quase ausência de atividade microbiológica, ocorrendo a incorporação dos compostos mais recalcitrantes de C por meio de degradação física e química do resíduo vegetal depositado (Franzluebbers et al., 1996).

Da mesma forma, o teor de C pode ser elevado, porém não complexado às frações minerais do solo (intra-agregados), tornando-se constantemente dependente do aporte de resíduos para sua manutenção, uma vez que durante o período de estiagem haveria aumento no teor do elemento no solo, enquanto no período com chuvas - por conseguinte, com atividade da microbiota - ocorreria a decomposição desses resíduos e a mineralização do C.

Assim, seriam justificáveis os baixos teores de C nos solos cultivados com mamona, independentemente do tempo de implantação da cultura (Quadro 1). Isso se deve, pois, conforme exposto anteriormente, nas áreas de mamona não há reposição dos resíduos vegetais da cultura. Os teores de $\mathrm{C}$ e $\mathrm{N}$ verificados nessas situações praticamente não modificam-se com o aumento da profundidade; dessa forma, o teor de $\mathrm{C}$ ou $\mathrm{N}$ verificado seria aquele ligado aos complexos organominerais da fração argila, protegido no interior dos agregados do solo.

Oliveira et al. (2008) verificaram que as camadas superficiais do solo são mais sensíveis às variações no 
C, devido à ação dos microrganismos na MOS. Assis et al. (2006) sustentam que os teores de C tendem a diminuir com o cultivo do solo quando comparado à vegetação nativa. Já Machado (2005) salienta que os teores de $\mathrm{C}$ superficiais tendem a diminuir, havendo pouca alteração em profundidades maiores.

A maior relação C: $\mathrm{N}$ foi encontrada nos solos de Caatinga (Quadro 1), certamente devido ao maior aporte de resíduos vegetais no solo. As demais situações cultivadas com mamona mantiveram seus teores de $\mathrm{C}$ e N muito similares, independentemente do tempo de implantação da cultura; portanto, a relação C: N também não se alterou.

Os estoques de $\mathrm{C}$ e $\mathrm{N}$ na Caatinga superam as demais situações com mamona em todas as camadas de solo (Quadro 1). Entre as áreas cultivadas com mamona, os diferentes tempos de implantação da cultura (10, 20 e 50 anos) não apresentaram diferença significativa nos estoques de $\mathrm{C}$ e $\mathrm{N}$ para as camadas amostradas.

Os estoques médios de $\mathrm{C}$ e $\mathrm{N}$ (Figura 1) para a camada de 0-30 cm neste estudo estão próximos aos verificados por Rossato et al. (2007) em estudo realizado na transição do bioma Caatinga para o Cerrado na região central da Bahia, com $86 \mathrm{Mg}_{\text {ha }}{ }^{-1}$ na área de vegetação nativa contra $54 \mathrm{Mg} \mathrm{ha}^{-1}$ na área cultivada com milho semeada manualmente sem a utilização de qualquer insumo agrícola. No mesmo estudo, esses autores encontraram estoques de $\mathrm{N}$ de 6 e $9 \mathrm{Mg} \mathrm{ha}^{-1}$ na área cultivada com milho e na vegetação nativa, respectivamente.

Pinheiro (2007), ao determinar as variações nos estoques de C no solo com cana-de-açúcar no semiárido, encontrou valores de $50 \mathrm{Mg}_{\text {ha }}{ }^{-1}$ nos tratamentos de cana sem queima contra $100 \mathrm{Mg} \mathrm{ha}^{-1}$ na vegetação nativa na Bahia para a camada de $30 \mathrm{~cm}$. Esse autor ainda destaca a importância, em termos de produtividade agronômica, dos elevados teores de $\mathrm{C}$ nesses solos.

$\mathrm{Na}$ figura 2 estão apresentadas as regressões exponenciais de decaimento dos estoques de $\mathrm{C}$ e N. A meia-vida da MOS foi estimada em 4,7 anos, ou seja, nesse intervalo de tempo, a mineralização da MOS promoveria redução nos estoques de $\mathrm{C}$ e $\mathrm{N}$ em $50 \%$.

Quadro 1. Densidade do solo, teores e estoques de C e N do solo para as situações sob vegetação nativa Caatinga e diferentes tempos de cultivo da mamona (10, 20 e 50 anos) na Fazenda Floresta, no município de Irecê (BA)

\begin{tabular}{|c|c|c|c|c|c|c|}
\hline \multirow{2}{*}{ Camada } & \multirow{2}{*}{ Densidade do solo } & \multicolumn{2}{|c|}{ Teor } & \multirow{2}{*}{ Razão C:N } & \multicolumn{2}{|c|}{ Estoque $^{(1)}$} \\
\hline & & $\mathbf{C}$ & $\mathbf{N}$ & & C & $\mathbf{N}$ \\
\hline \multirow[t]{2}{*}{$\mathrm{cm}$} & $\mathrm{g} \mathrm{cm}^{-3}$ & \multicolumn{2}{|c|}{$\mathrm{g} \mathrm{kg}^{-1}$} & & \multicolumn{2}{|c|}{$\mathrm{Mg} \mathrm{ha}^{-1}$} \\
\hline & \multicolumn{6}{|c|}{ Caatinga } \\
\hline $0-5$ & $1,14 \mathrm{aA}$ & $51,7 \mathrm{aA}$ & $4,2 \mathrm{aA}$ & $12,3 \mathrm{aA}$ & $22,68 \mathrm{~A}$ & $1,84 \mathrm{~A}$ \\
\hline $5-10$ & $1,02 \mathrm{bBC}$ & $35,6 \mathrm{bA}$ & $3,3 \mathrm{bA}$ & $10,8 \mathrm{aA}$ & $18,44 \mathrm{~A}$ & $1,67 \mathrm{~A}$ \\
\hline $10-20$ & $0,95 \mathrm{bcB}$ & $26,2 \mathrm{cA}$ & $3,4 \mathrm{bA}$ & $7,7 \mathrm{bB}$ & $28,04 \mathrm{~A}$ & $3,57 \mathrm{~A}$ \\
\hline \multirow[t]{2}{*}{$20-30$} & $0,97 \mathrm{bB}$ & $18,3 \mathrm{dA}$ & $3,5 \mathrm{bA}$ & $5,2 \mathrm{bB}$ & $18,87 \mathrm{~A}$ & $3,61 \mathrm{~A}$ \\
\hline & \multicolumn{6}{|c|}{ Mamona 10 anos } \\
\hline $0-5$ & $0,99 \mathrm{cB}$ & $15,3 \mathrm{aB}$ & $1,9 \mathrm{aB}$ & $8,1 \mathrm{aB}$ & $7,80 \mathrm{~B}$ & $0,97 \mathrm{~B}$ \\
\hline $5-10$ & $1,28 \mathrm{aA}$ & $15,1 \mathrm{aB}$ & $1,8 \mathrm{aB}$ & $8,3 \mathrm{aB}$ & $5,90 \mathrm{~B}$ & $0,70 \mathrm{BC}$ \\
\hline $10-20$ & $1,24 \mathrm{aA}$ & $13,7 \mathrm{aB}$ & $1,7 \mathrm{aB}$ & $8,1 \mathrm{aB}$ & $13,93 \mathrm{~B}$ & $1,72 \mathrm{~B}$ \\
\hline \multirow[t]{2}{*}{$20-30$} & $1,13 \mathrm{bA}$ & $12,6 \mathrm{bB}$ & $1,6 \mathrm{aB}$ & $7,8 \mathrm{aA}$ & $12,38 \mathrm{~B}$ & $1,57 \mathrm{~B}$ \\
\hline & \multicolumn{6}{|c|}{ Mamona 20 anos } \\
\hline $0-5$ & $1,07 \mathrm{bAB}$ & $13,9 \mathrm{aB}$ & $1,7 \mathrm{aB}$ & $8,2 \mathrm{aB}$ & $6,50 \mathrm{~B}$ & $0,79 \mathrm{BC}$ \\
\hline $5-10$ & $0,81 \mathrm{cC}$ & $14,1 \mathrm{aB}$ & $1,7 \mathrm{aB}$ & $8,3 \mathrm{aB}$ & $8,71 \mathrm{~B}$ & $1,05 \mathrm{~B}$ \\
\hline $10-20$ & $1,27 \mathrm{aA}$ & $14,3 \mathrm{aB}$ & $1,7 \mathrm{aB}$ & $8,4 \mathrm{aB}$ & $11,26 \mathrm{~B}$ & $1,34 \mathrm{~B}$ \\
\hline \multirow[t]{2}{*}{$20-30$} & $1,04 \mathrm{bB}$ & $12,5 \mathrm{bB}$ & $1,6 \mathrm{aB}$ & $7,8 \mathrm{aA}$ & $12,21 \mathrm{~B}$ & $1,55 \mathrm{~B}$ \\
\hline & \multicolumn{6}{|c|}{ Mamona 50 anos } \\
\hline $0-5$ & $1,17 \mathrm{aA}$ & $15,0 \mathrm{aB}$ & $1,6 \mathrm{aB}$ & $9,3 \mathrm{aA}$ & $6,41 \mathrm{~B}$ & $0,68 \mathrm{C}$ \\
\hline $5-10$ & $1,18 \mathrm{aB}$ & $15,0 \mathrm{aB}$ & $1,7 \mathrm{aB}$ & $8,8 \mathrm{aB}$ & $7,45 \mathrm{~B}$ & $0,84 \mathrm{BC}$ \\
\hline $10-20$ & $1,22 \mathrm{aA}$ & $14,4 \mathrm{aB}$ & $1,6 \mathrm{aB}$ & $9,0 \mathrm{aA}$ & $11,86 \mathrm{~B}$ & $1,32 \mathrm{~B}$ \\
\hline $20-30$ & $1,12 \mathrm{bA}$ & $11,9 \mathrm{bB}$ & $1,4 \mathrm{aB}$ & $8,5 \mathrm{aA}$ & $12,30 \mathrm{~B}$ & $1,43 \mathrm{~B}$ \\
\hline
\end{tabular}

${ }^{(1)}$ Estoque = estoque de $\mathrm{C}$ ou $\mathrm{N}$ corrigido pela massa de solo equivalente para a densidade de $1 \mathrm{~g} \mathrm{~cm}^{-3}$. Médias seguidas de mesma letra, minúsculas nas camadas e maiúsculas entre as situações amostradas na mesma camada, não diferem estatisticamente pelo teste de SNK $(p<0,05)$. 
Com a equação exponencial de decaimento, estimouse que no $14^{\circ}$ ano após a conversão das áreas a perda de MOS passou a ser inferior a $0,5 \%$ ano $^{-1}$, o que poderia ser considerado um período de estabilização nas perdas de $\mathrm{C}$ do solo.

As variações nos estoques de $\mathrm{C}$ e $\mathrm{N}$ mostraram que as maiores perdas ocorreram nos primeiros anos após a conversão da vegetação nativa de Caatinga para a situação Mamona 10 anos, com um fator de emissão de C calculado em 4,8 Mg C $\mathrm{ano}^{-1}$ (Figura 2). Contudo, o método proposto no volume 4 do Guia para Inventários Nacionais de Gases de Efeito Estufa (IPCC, 2006) recomenda a utilização de 20 anos para esse tipo de avaliação, e, uma vez que este estudo oferece essa oportunidade, o fator de emissão para este período (Mamona 20 anos) foi estimado em $2,47 \mathrm{Mg} \mathrm{C}_{\text {ano }}{ }^{-1}$.

Por não haver utilização de nenhum insumo, reposição de fertilizantes minerais ou aporte de resíduos vegetais nas situações cultivadas com mamona, pode-se inferir que, após o período de intensa perda de $\mathrm{C}$ (mais de 20 anos), não ocorreu a recuperação dos estoques de $\mathrm{C}$ no solo até o presente momento. Dessa forma, o sistema permaneceu em um novo equilíbrio ao longo dos anos de cultivo.
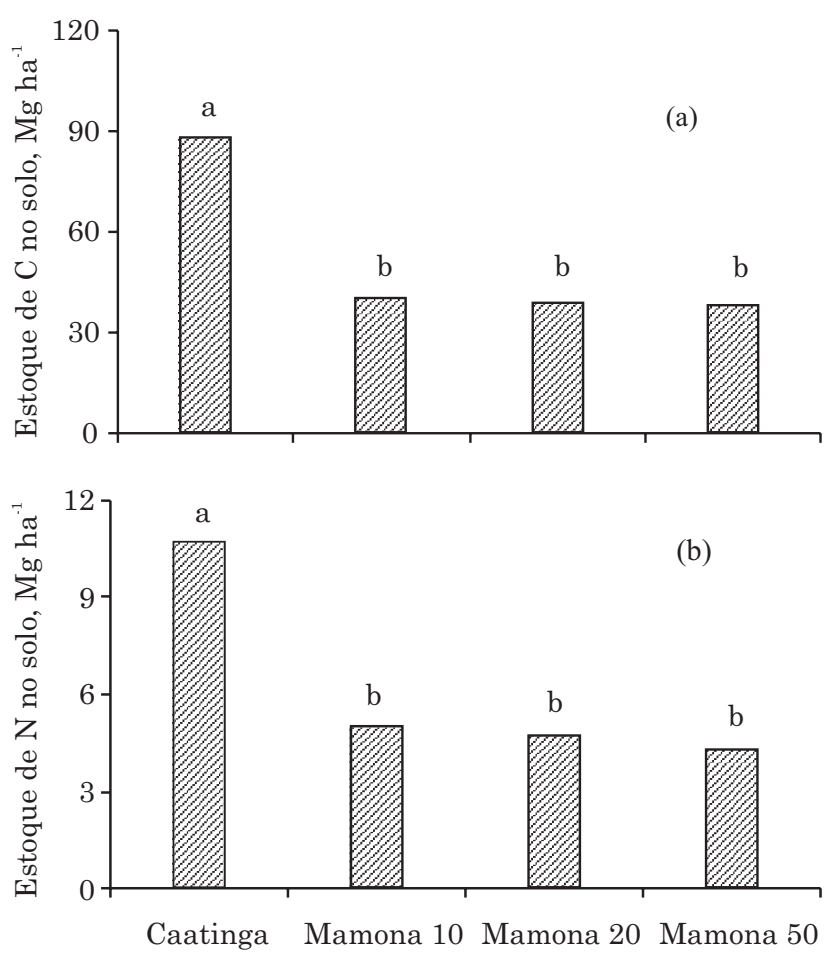

Figura 1. Estoques de carbono (A) e nitrogênio (B) do solo $\left(\mathrm{Mg} \mathrm{ha}^{-1}\right)$ para a camada de 0-30 cm nas situações sob vegetação nativa de Caatinga $e$ diferentes tempos de cultivo da mamona $(10,20$ e 50 anos) na Fazenda Floresta, no município de Irecê (BA). Letras não diferem estatisticamente pelo teste de SNK $(p<0,05)$.

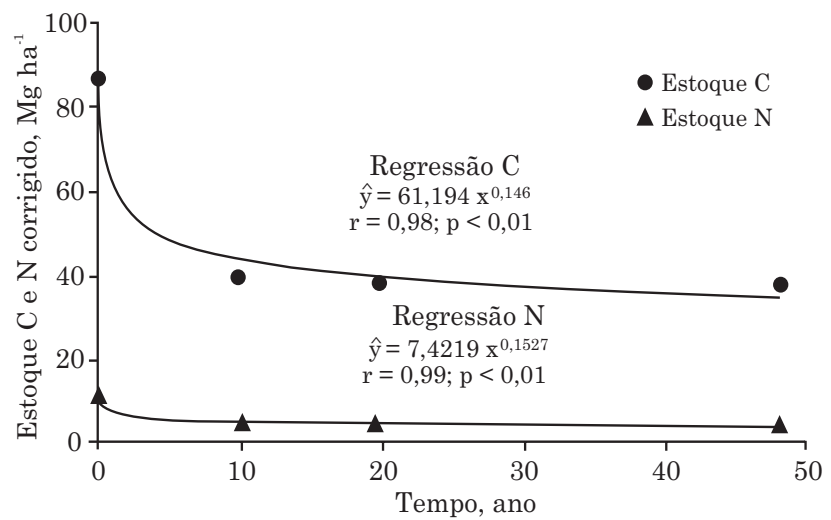

Figura 2. Regressão da variação dos estoques de $\mathrm{C}$ e $\mathrm{N}$ no solo para a camada de $0-30 \mathrm{~cm}$ entre a vegetação natural - Caatinga $($ tempo $=0)$ e as situações com diferentes tempos de cultivo da mamona (10, 20 e 50 anos) na Fazenda Floresta, no município de Irecê (BA).

\section{CONCLUSÕES}

1. A conversão da caatinga para o cultivo da mamona ocasiona diminuição de cerca de $50 \%$ no estoque de $\mathrm{C}$ e $\mathrm{N}$ no solo.

2. A meia-vida da MOS calculada para a região do semiárido estudada é de 4,7 anos.

3. O fator de emissão de $\mathrm{C}$ do solo, devido à mudança de uso do solo após 20 anos, conforme proposto pelo IPCC, é de $2,47 \mathrm{Mg} \mathrm{C}$ ano-1.

\section{AGRADECIMENTOS}

Aos proprietários da Fazenda Floresta, srs. Jairo e Vital Dourado, pela contribuição na execução do trabalho. À Petrobras, pelo financiamento do projeto.

\section{LITERATURA CITADA}

ASSIS, C.P.; OLIVEIRA, T.S.; DANTAS, J.A.N. \& MENDONÇA, E.S. Organic matter and phosphorus fractions in irrigated agroecosystems in a semi-arid region of Northeastern Brazil. Agric. Ecosyst. Environ., 138:7482,2010 .

ASSIS, C.P.; JUCKSCH, I.; MENDONÇA, S. \& NEVES, J.C.L. Carbono e nitrogênio em agregados de Latossolo submetido a diferentes sistemas de uso e manejo. Pesq. Agropec. Bras., 41:1541-1550, 2006.

BERNOUX, M.; ARROUAYS, D.; CERRI, C.C. \& BOURENNANE, H. Modelling vertical distribution of carbon in Oxisols of the Western Brazilian Amazon (Rondônia). Soil Sci., 163:941-951, 1998. 
BERNUOX, M.; CARVALHO, M.C.S.; VOLKOFF, B. \& CERRI, C.C. Brazil's soil carbon stocks. Soil Sci. Soc. Am. J., 66:888-896, 2002.

BLAKE, G.R. \& HARTGE, K.H. Bulk density. In: KLUTE, A., ed. Methods of soil analysis. 2.ed. Madison, ASA, 1986. p.363-375. (Agronomy Monograph, 9)

BRASIL. Ministério do Meio Ambiente. Avaliações e ações prioritárias pra conservação da biodiversidade da caatinga. Brasília, 2002.

CERRI, C.C.; MAIA, S.M.F.; GALDOS, M.V.; CERRI, C.E.P.; FEIGL, B.J. \& BERNOUX, M. Brazilian greenhouse gas emissions: the importance of agriculture and livestock. Sci. Agric., 66:831-843, 2009.

CÉSAR, A.S. \& BATALHA, M.O. Biodiesel production from castor oil in Brazil: A difficult reality. Energy Policy, 38:4031-4039, 2010.

EMPRESA BRASILEIRA DE PESQUISA AGROPECUÁRIA EMBRAPA. Relatório: biocombustíveis, 2007. Disponível em: <http://www.embrapa.br>. Acesso em: 10 de out. de 2009.

EMPRESA BRASILEIRA DE PESQUISA AGROPECUÁRIA EMBRAPA. Sistema brasileiro de classificação de solos. 2.ed. Rio de Janeiro, 2006. 306p.

FRANZLUEBBERS, A.J.; HANEY, R.L.; HOND, F.M. \& ZUBERER, D.A. Determination of microbial biomass and nitrogen mineralization following rewetting of dried soils. Soil Sci. Soc. Am. J., 60:1133-1139, 1996.

FRAZÃO, L.A.; SANTANA, I.K.S.; CAMPOS, D.V.B.; FEIGL, B.J. \& CERRI, C.C. Estoques de carbono e nitrogênio e fração leve da matéria orgânica em Neossolo Quartzarênico sob uso agrícola. Pesq. Agropec. Bras., 45:1198-1204, 2010.

FREITAS, S.M. \& FREDO, C.E. Biodiesel a base de óleo de mamona: Algumas considerações. Inf. Econ., 35:37-42, 2005.

GALVÃO, S.R.S.; SALCEDO, I.H. \& SANTOS, A.C. Frações de carbono e nitrogênio em função da textura, do relevo e do uso do solo na microbacia do agreste em Vaca Brava (PB). R. Bras. Ci. Solo, 29:955-962, 2005.

INSTITUTO BRASILEIRO DE GEOGRAFIA ESTATÍSTICA IBGE. Levantamento sistemático da produção agrícola 2010. Disponível em: <http://www.ibge.gov.br>. Acesso em: 01 de mar. de 2011.

INTERGOVERNMENTAL PANEL ON CLIMATE CHANGE IPCC. The physical science basis: Summary for policymakers. Contribution of working group I to the fourth assessment report of the intergovernmental panel on climate change. Cambridge, Cambridge University Press, 2006.

JANSEN, H.H. Soil carbon: A measure of ecosystem response in a changing world? Can. J. Sci., 85:467-480, 2005.

MACHADO, P.L.A. Carbono do solo e a mitigação da mudança climática global. Quím. Nova, 28:329-334, 2005.

MAIA, S.M.F.; OGLE, S.M.; CERRI, C.C. \& CERRI, C.E.P. Changes in soil organic carbon storage under different agricultural management systems in the Southwest Amazon Region of Brazil. Soil Tillage Res., 106:177-184, 2010.
MAIA, S.M.F.; OGLE, S.M.; CERRI, C.E.P. \& CERRI, C.C. Effect of grassland management on soil carbon sequestration in Rondônia and Mato Grosso states, Brazil. Geoderma, 149:84-91, 2009.

MAIA, S.M.F.; XAVIER, F.A.S.; OLIVEIRA, T.S.; MENDONÇA, E.S. \& ARAUJO FILHO, J.A. Impactos de sistemas agroflorestais e convencional sobre a qualidade do solo no semi-árido cearense. R. Árvore, 30:837-848, 2006.

MAIA, S.M.F.; XAVIER, F.A.S.; OLIVEIRA, T.S.; MENDONÇA, E.S. \& ARAUJO FILHO, J.A. Organic carbon pools in a Luvisol under agroforestry and conventional farming systems in the semi-arid region of Ceará, Brazil. Agrofor. Syst., 71:127-138, 2007.

MENEZES, R.S.C.; SALCEDO, I.H. \& ELLIOTT, E.T. Microclimate and nutrient dynamics in a silvopastoral system of semiarid northeastern Brazil. Agrofor. Syst., 56:27-38, 2002.

MENEZES, R.S.C. \& SAMPAIO, E.V.S.B. Simulação dos fluxos e balanços de fósforo em uma unidade de produção agrícola familiar no semi-árido paraibano. In: SILVEIRA, L.M.; PETERSEN, P. \& SABOURIN, E., orgs. Agricultura familiar e agroecologia no semi-árido: Avanços a partir do agreste da Paraíba. Rio de Janeiro, AS-PTA, 2002. p.249-260.

OLIVEIRA, J.T.; SANTOS, A.M.S.; MOREAU, A.M.; MENEZES, A.A. \& COSTA, O.V. Características físicas e carbono orgânico de solos sob diferentes tipos de uso da terra. R. Bras. Ci. Solo, 32:132-138, 2008.

PAES-SILVA, A.P. Cobertura vegetal da bacia hidrográfica do Açude do Namorado no Cariri Oriental Paraibano. Areia, Universidade Federal da Paraíba, 2002. 107p. (Tese de Mestrado)

PINHEIRO, E.F.M. Estoques de carbono e nitrogênio num Argissolo Amarelo cultivado com cana-de-açúcar: Influência da queima ou manutenção da palhada. Seropédica, Embrapa Agrobiologia, 2007. 21p.

ROSSATO, M. Recursos genéticos de palmeiras nativas do gênero butia do Rio Grande do Sul. Pelotas, Universidade Federal de Pelotas, 2007. 136p. (Tese de Doutorado)

SAMPAIO, E.V.S.B. \& SALCEDO, I.H. Diretrizes para o manejo sustentável dos solos brasileiros: região semiárida. In. CONGRESSO BRASILEIRO DE CIÊNCIA DO SOLO, 26., Mesa Redonda. Anais... Rio de Janeiro, 1997.

SAS. Institute. SAS/STAT guide for personal computer: Version 9.1 Cary, 2003.

SAVY FILHO, A. \& BANZATTO, N.V. O mercado está para a mamona. Casa Agric., 5:12-15, 1983.

SEVERINO, L.S.; MORAES, C.R.A.; GONDIM, T.M.S.; CARDOSO, G.D. \& SANTOS, J.W. Fatores de conversão do peso de cachos e frutos para peso de sementes de mamona. Campina Grande, Embrapa Algodão, 2004. 15p. 
SIQUEIRA NETO, M.; PICCOLO, M.C.; SCOPEL, E.; COSTA JUNIOR, C.; CERRI, C.C. \& BERNOUX, M. Carbono total e atributos químicos com diferentes usos do solo no Cerrado. Acta Sci. Agron., 34:709-717, 2009.

SIQUEIRA NETO, M.; SCOPEL, E.; CORBEELS, M.; CARDOSO, A.N.; DOUZET, J.M.; FELLER, C.; PICCOLO, M.C.; CERRI, C.C. \& BERNOUX, M. Soil carbon stocks under no-tillage mulch-based cropping systems in the Brazilian Cerrado: An on-farm synchronic assessment. Soil Tillage Res., 110:187-195, 2010.

SISTI, C.P.J.; SANTOS, H.P.; KOHHANN, R.; ALVES, B.J.R.; URQUIAGA, S. \& BODDEY, R.M. Change in carbon and nitrogen stocks in soil under 13 years of conventional or zero tillage in Southern Brazil. Soil Tillage Res., 76:39-58, 2004.
SOUSA, F.P.; FERREIRA, T.O.; MENDONÇA, E.S.; ROMERO, R.E. \& OLIVEIRA, J.G.B. Carbon and nitrogen in degraded Brazilian semi-arid soils undergoing desertification. Agric. Ecosyst. Environ., 148:11-21, 2012.

STRECK, C.A.; REINERT, D.J.; REICHERT, J.M. \& KAISER, D.R. Modificações em propriedades físicas com a compactação do solo causada pelo tráfego induzido de um trator em plantio direto. Ci. Rural, 34:755-760, 2004.

XAVIER, F.A.S.; MAIA, S.M.F.; OLIVEIRA, T.S. \& MENDONÇA, E.S. Biomassa microbiana e matéria orgânica leve em solos sob sistemas agrícolas orgânico e convencional na Chapada da Ibiapaba - CE. R. Bras. Ci. Solo, 30:247-258, 2006. 